\title{
Astronomical exploration and the public imagination
}

\author{
Baruch S. Blumberg \\ NASA Lunar Science Institute and NASA Astrobiology Institute, \\ NASA Ames Research Center, Moffett Field, California 94035, USA \\ email: Baruch.Blumberg@fccc.edu
}

\section{Introduction}

Humans have a need to understand where they fit in the cosmos. Driven by the unlimited possibilities of human imagination the night sky has been and is one of the most powerful stimulators of curiosity. In pre-modern times, farmers, pastoralists, travelers, even city dwellers unhampered by light pollution, had many opportunities to observe and wonder on the mysteries of the starry night. In this, the International Year of Astronomy marking the 400th anniversary of Galileo's telescopic observations (that is also the 200th anniversary of the birth of Charles Darwin) there are many explorations using the advanced and expensive instruments that society provides for satisfying the public curiosity and, of course, that of the astronomers trained to ask and answer the questions. However, it is a truism that scientific answers always raise new questions that could not have been asked raised prior to the preceding answers. The more we know the more we know about what we do not know; the task of scientific inquiry, or, for that matter, inquiry in general, is endless.

The questions touch on matters of fundamental interest; how did the cosmos begin, what happened before the beginning, if anything, how did life begin, and how did sentient beings, such as humans, evolve to devise the instruments, make the observations, compose the theories, answer the questions and raise many others.

Parallel with astronomical advances, biological research has progressed rapidly. It can now more readily investigate the deep history of origins, including the history of contemporary genomes using the information contained within the genome itself as well as in fossils. Analogous to the great distances outward observed by astronomers to study origins, biologists can look inward at small dimensions to tell the story of how inorganic matter, rocks, patterns, and energy became biology.

I am not an astronomer; my training has been in medicine and biochemistry. My acquaintance with astronomy came from my rôle as the former Director of the NASA Astrobiology Institute. In the spirit of the theme of this Symposium, astronomy in society and culture, I have been asked to speak on astronomy and the public imagination and I will briefly address several topics that relate to this issue.

\section{Human and robots in space research}

The astronomical and space research process raises the question of what it means to be human. In the area of space exploration, at a practical level, should space missions be robotic or human? There is a basic human need to explore and to go beyond their planet of origin. Space is a continuation of the human quest to seek the mysterious and unknown. Human missions are much more expensive and dangerous than robotic exploration. Are humans needed for exploration? Are there limitations to robotic possibilities? Can a robot 


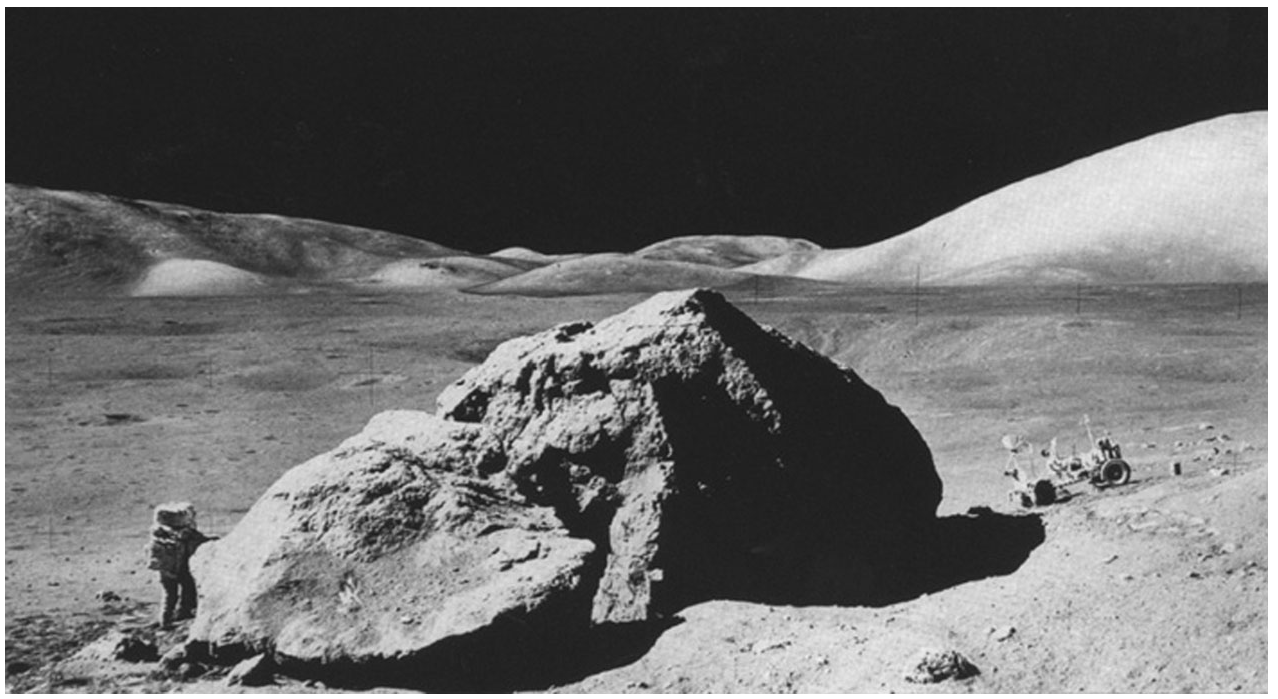

Figure 1. Harrison Schmidt, Ph.D., last human on the Moon.

be programmed to select, see and analyse phenomena that have never been previously experienced and for which they were not and could not be programmed?

Harrison Schmidt, the geologist who, along with astronaut Eugene Cernan, spent 75 hours on the Moon during the Apollo 17 mission (17-19 December 1972), expressed this eloquently in his article "Field Trip to The Moon" $\dagger$ :

The third EVA provided an opportunity to study the large boulders that had rolled and bounced down the north wall of the valley. From them, we hoped to learn more about what happens when large objects from space hit, break, and partially melt planetary crusts. During the detailed examination of one very large boulder, the unexpected discovery of a subtle contact between two types of impact generated debris units, one intrusive into the other, again proved the worth of the trained human eye in exploration. Questions often arise as to whether robotic exploration of the Moon or any other planet would be less expensive than human exploration and provide all the essential scientific return. This question, of course, can never be answered to everyone's satisfaction if only because of sincere disagreements over what constitutes "essential science". Clearly, robotic systems will and must make increasingly important contributions, however, the spontaneous human observation, integration, and interpretation of the total dynamic situation involved in space activities, and a calculated human response to that situation, will be as irreplaceable in the future as throughout the past.

Schmidt's argument appears to be that there is a human need for exploration and discovery, forged by human imagination that is not possible with programmed or selflearning robots. The personality of HAL, the computer in Stanley Kubrick's and the late Arthur Clarke's 1968 masterpiece movie (and later novel), "2001: A Space Odyssey" puts a fascinating take on the question. It posits a deadly contest between the wills of the very intelligent computer and the human crew $\ddagger$. HAL was programmed to complete a successful mission and could not appreciate nuanced deviations from this goal that had to be altered by events that took place during the trip. Would a human decision maker

$\dagger$ fti.neep.wisc.edu/neep602/LEC1/trip.html

$\ddagger$ HAL's name was derived from the acronym for Heuristically programmed ALgorithmic Computer, not as a one letter downward removed from IBM, the initials of the International Business Machines company, as is popularly supposed. 


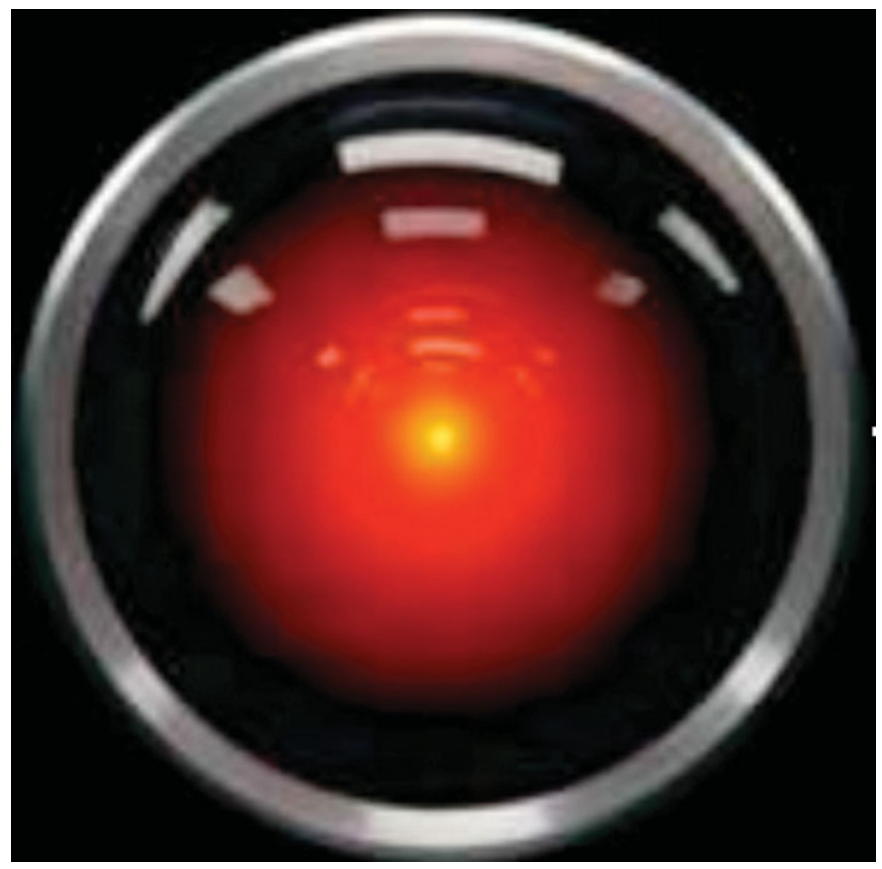

Figure 2. HAL's iconic Camera Eye from Kubrick and Clarke "2001 A Space Odyssey". HAL stands for Heuristically programmed ALgorithmic computer.

have decided differently influenced by prior experience, emotion, and a more complex approach to the problem than had HAL?

\section{Robotic and humans on missions}

Human and machine interactions also arises in an interesting fashion for the indomitable Mars Exploration Rovers (MERs) now approaching the fifth year of a mission planned for 90 days! (MER Spirit landed on Mars on January 4, 2004 and Opportunity on January 24, 2004.)

Utilising sets of stereoscopic cameras on a mast and at platform level they have a measure of autonomous obstacle avoidance capability including planning for avoidance of objects a moderate distances ahead. They can engage in tactics; they do not peer into the middle and far distance and decide what the next target for scientific exploration should be. The science and engineering teams at the Jet Propulsion Laboratory over a hundred million kilometers away provided the long-range planning, the strategy for the rovers. A particularly exciting image demonstrating the possibilities of machine-machine and machine-human interaction is the picture of the MER Opportunity at the edge of the Victoria impact crater on Mars.

The decision was being weighed of descending into the crater to explore the exposed layers on the walls of the crater and the fascinating dunes within it. Images of MER Opportunity and its surroundings were taken by the Mars Reconnaissance Orbiter and relayed to the control center at Jet Propulsion Laboratory (JPL) in Pasadena, California. There was a cooperative program between the rover, the orbiter, and the engineering and science teams at JPL. The science team and not the robot itself made the decision. But, over time, the machines developed a personality, perhaps not as advanced as that of HAL, but also not as sinister. AI and AT experts assure us that human-like decision making 


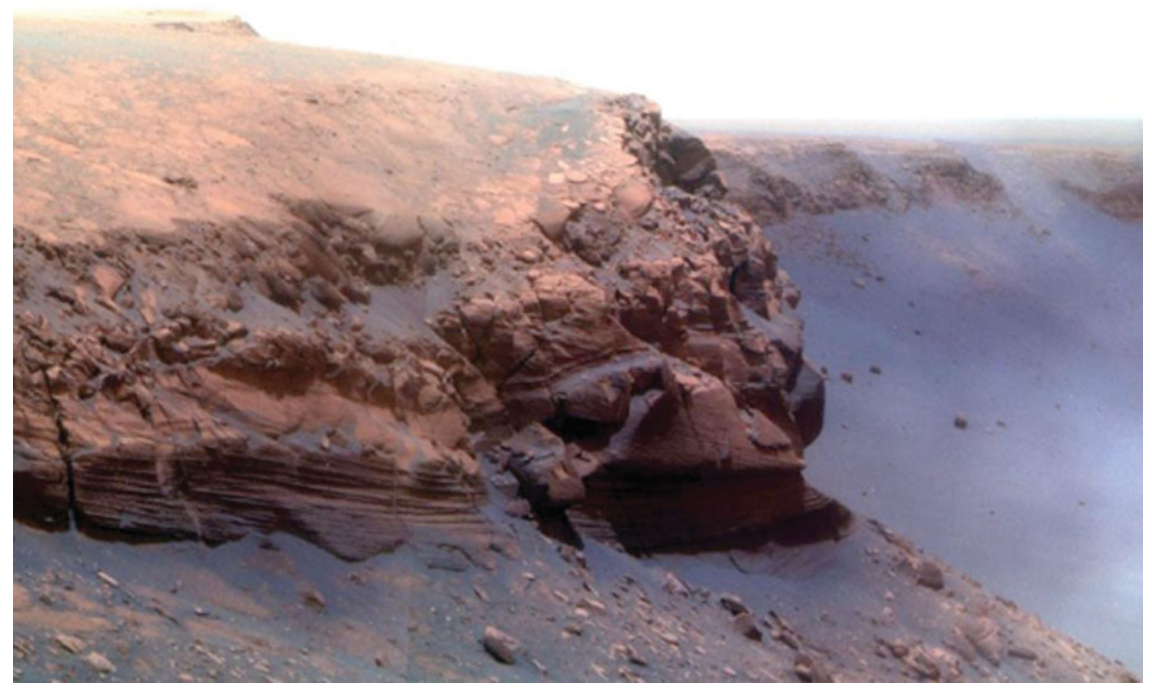

Figure 3. Cape Verde, Victoria crater, Mars.

will be assignable to robots in the future, particularly when the time of convergence occurs. Convergence is when the speed and capabilities of computers reach and then exceed those of humans; time will tell if this is the case.

Opportunity conducted its studies of the Victoria Crater for a year. Its explorations included the base of Cape Verde cliff allowing the study of geological layering at a considerable depth. Opportunity climbed out of Victoria and is now on its way to an even larger impact crater.

Human-machine interactions are becoming more and more a consideration in the practice of science and of astronomy in particular. It is no longer necessary for the observer to sit by the telescope on a mountaintop, cold and exposed to the effects of low atmospheric pressure.

The images are collected digitally and transmitted electronically to a digital database that is accessible via the web. The astronomer can sit with his or her laptop, for example, at a surfing beach in Australia, and make observations from a presumably more comfortable non-mountaintop environment.

Many scientific instruments can now collect and store data 24/7. Star surveys assemble tera bites of information and store them in data banks that can be mined by the scientists at a long remove from the observing telescope. The ability to handle large amounts of data has moved science forward in a way that was impossible in the pre-data base management days. However, will their use, removed from the context from where the observations were made, decrease the possibility of chance or serendipitous observations? Does the scientist learn something that contributes to intuitive knowledge, not easily definable, when closer to the source of the data? Independent of the answer to these questions these techniques have been central to the massive increase in recent years in knowledge and understanding.

In a recent Nature (1 January 2009) several issues relating to astronomy and human imagination were discussed. It is an amazing fact that about $20 \%$ of the human population can no longer see the Milky Way because of the light pollution associated with cities, and 


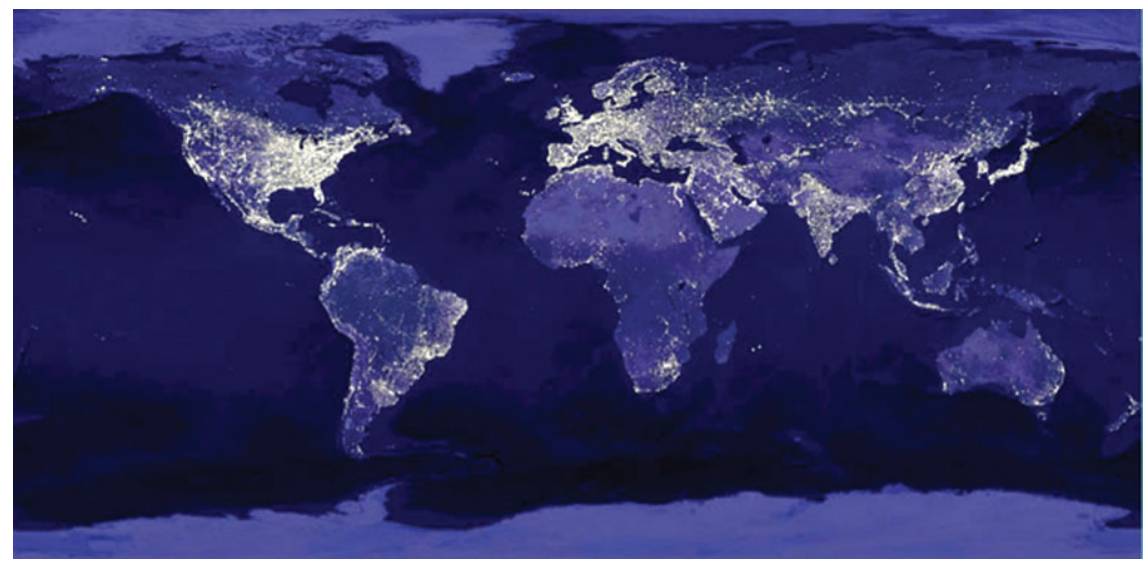

Figure 4. The world at night (light has been enhanced).

the increasing percentage of the population who reside in cities. As urbanisation and the availability of electric power increases larger portions of the planet will be come polluted.

Will this diminish the imaginative inspiration of the directly observed sky? There is an interest in setting aside Earth dark places, the equivalent of parks, where darkness suitable for astronomic observations, or for human experiences with the night sky undiminished by extraneous light. An argument for the establishment of research stations on the Moon would be to providing darkness for telescope placement and, possibly, for the enjoyment of touring space travelers. There is a similar argument for placing radio telescopes on the side of the Moon shielded from Earth. They could receive radio signal undisturbed by radiofrequency pollution from Earth, including signals from extraterrestrial cultures, if they exist, that may be trying to communicate. (Apparently, the Earth has now less radio frequency pollution since much of the transmission is by fiber optic cable.)

The next planned human mission that is likely to stir the human imagination is to the Moon to establish a continuously inhabited research field station. The NASA Lunar Institute (NSLI) at NASA Ames Research Center, Moffett Field, California, was established last year to fund science associated with the Moon exploration. The Moon exploration programs are international; China, India and other countries have programs planned and active. I am on the staff, part time, of the NLSI.

Biological and astronomical research come together in astrobiology programs. The NASA Astrobiology Institute, established in 1998, funds about dozen research teams and there are similar institutions internationally.

\section{The Hubble Deep Field and deep exploration of the genome}

The iconic Hubble Deep Field image (taken September 24, 2003 to January 16, 2004) is a remarkable presentation of the vastness of space time. The Big Bang is estimated to have happened 13.7 billion years ago. The oldest objects seen in this tunnel down time and space originated from 0.4 to 0.7 billion years after the Big Bang. The distances and antiquity are difficult to comprehend on our ordinary terrestrial scale. This image, and similar cosmic pictures that are now available nearly daily from orbiting- and Earthbased telescopes are one of the greatest igniters of the imagination and have surely been the inspiration for many science fiction stories. 


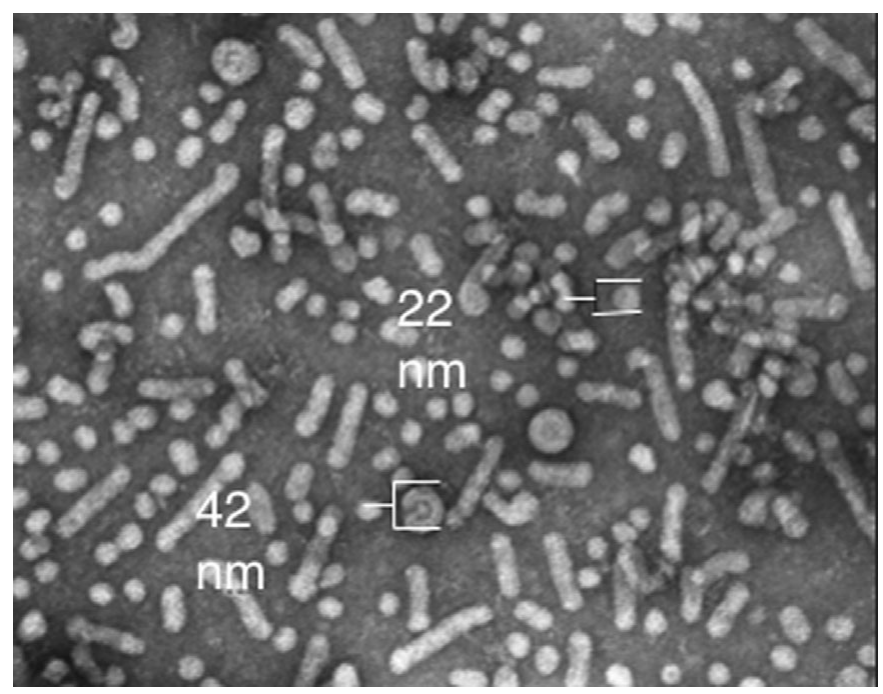

Figure 5. Hepatitis B virus morphology. Characteristics: -Nucleic acid: DNA; -Classification: Hepadnavirus type 1; -Serotypes: multiple; -In vivo replication: Reverse transcription in liver and other tissues; - In vitro propagation: Primary hepatocyte culture and transfection by cloned HBV DNA.

Looking in the opposite direction in the dimensional scale, compare these dimensions with those of a virus visualised through an electron microscope.

The hepatitis B virus has been the subject of my research since 1967 . It is one of the most common pathogenic viruses. HBV has infected about one third of the world's population; most resist the infection and develop protective antibodies against it, but nearly 400 million remain infected. They are at risk of developing acute hepatitis, or remain chronically infected and are at risk of succumbing to life shortening chronic hepatitis, or cancer of the liver that is very difficult to treat. Fortunately, the vaccines derived from our invention in 1969 are now widely used; more than 70\% of the members of the WHO now have national vaccination programs and the incidence of infection and primary cancer of the liver is decreasing dramatically.

The virus itself is 42 nanometers in diameter and contained within it is the information on the DNA of the virus. The smaller particles that are made up entirely of the surface coating of the virus are 22 nanometers, and the elongated particles are 22 nanometers wide and of variable lengths. Looking down the space-time tunnel of the Ultra Deep Field provides knowledge of the origins of the cosmos. Similarly, probing the genome of the virus reveals information about evolutionary relations and connections with other organisms, including relations that are remote in time. Continuing study could approach the time of origin of DNA and living matter itself. Genomes are conserved; that is, there are strong similarities (homologies) between the DNA sequences of members of one species and those to which it is closely and even remotely related on the evolutionary scale. A species carries its evolutionary history within its genome.

The genome may often be the repository of fossils analogous to the preservation of fossils in geologic sedimentary formations. Following infection, retroviruses, a class of viruses, can insert DNA sequences related to their RNA into the genome of the host and reside there indefinitely. If they enter germ-line DNA - the DNA within the egg or sperm- they can be inherited and are similar to an insertional mutation, but with many more genome changes. These sequences are called endogenous retroviruses (ERVs); the term is somewhat misleading in that they are not independent infectious organisms. ERV 
sequences can make up about $8 \%$ of the human genome. This is larger than the sequences that make up the reading frames, that is, the sections of the genome that produce the proteins that make up much of the body and are important functional elements of life. Some of these viruses entered contemporary genomes many years ago and no longer exist as entities outside of a host genome; they are disappeared species. They can, in effect, be extracted from the host genome to examine their relation to contemporary forms. A retrovirus, similar to HIV, the modern virus that causes AIDS, was extracted from a lemur that is only found in Madagascar and identified identified as a virus that has contributed to the genome of the contemporary human HIV virus. This provides information on the relation of lemur to other species, including humans, and provides a piece of early history of the viruses that infect prosimians and the contemporary virus that is the cause of the terrible human epidemic that now affects many humans.

Astronomers can look up enormous distances into space-time to try to understand cosmic origins. Biologists can look the other way at very small dimensions deep into the genomes of living species and learn about life's early history and, possibly, its origins.

\section{Public participation in astronomy and science}

Continued research in astronomy, and in all basic science, requires funding by the government and the public. How can their interest and support be maintained and their imaginations nourished? An effective method is to have the public participate in science. There are many programs for citizen science that is for having non-professional volunteers take part in the collection of data, analysis, and participation in the whole process of scientific exploration. Professional astronomers have worked very closely with amateurs. For example, the Spaceguard program combines the work of professional astronomers, government, and citizen astronomer volunteers to search for and predict the orbits of near Earth objects that might impact the Earth. There is considerable interest in these programs as the possibility of intervention to deflect impact is under consideration. There are many other programs including Galaxy Zoo and Moon Zoo in which amateur volunteers classify galaxies and characterise Moon craters and surface features.

Science and particularly astronomy have the capacity to encourage the imagination of scientists and others and to help move forward the enterprise of understanding our natural world. 